\title{
IMPORTANCE OF SPERM CAPACITATION FOR GAMETE CONTACT IN THE RABBIT
}

\author{
J. W. OVERSTREET AND J. M. BEDFORD*
}

Department of Anatomy and International Institute for the Study of Human Reproduction, Columbia University, College of Physicians and Surgeons, New York, N.Y. 10032, U.S.A.

(Received 30th Fanuary 1974)

The capacitation process has been suggested as a prerequisite for every stage of gamete interaction, including penetration of the granulosa cell investment (Austin, 1960), contact with the zona pellucida (Bedford, 1967; Hartmann \& Gwatkin, 1971), penetration of the zona substance (Bedford, 1970) and, for the hamster at least, incorporation into the vitellus (Yanagimachi \& Noda, 1970).

Morphological studies have demonstrated a characteristic fusion and vesiculation of the plasma membrane and outer acrosomal membrane of the spermatozoon during its passage through the granulosa cell mass (Barros, Bedford, Franklin \& Austin, 1967). Capacitation is considered to be a prerequisite for this acrosome reaction and it may be inferred that membrane changes are related to capacitation as well (Bedford, 1969, 1970). It is uncertain, however, whether the initial contact between the rabbit spermatozoon and the zona pellucida is facilitated by alteration of the sperm surface (Bedford, 1967; Thibault, 1971), or whether this interaction is merely a sequel to the passage of the spermatozoon through the granulosa cell investment.

In the present experiments, the abilities of capacitated and non-capacitated spermatozoa to approach and adhere to the zona pellucida have been compared directly following intratubal insemination of a mixture of the two populations. In this competitive assay system, the population identity of the spermatozoa associated with the egg could be differentiated by the presence or absence of a fluorescent label (Mellish \& Baker, 1970; Overstreet \& Adams, 1971; Baker $\&$ Degen, 1972). In a second study, the ability of non-capacitated spermatozoa to traverse the corona radiata was investigated. Denuded and corona-invested eggs were transferred to oviducts containing capacitated or non-capacitated spermatozoa and one of the egg populations was labelled with a fluorescent dye (Overstreet, 1973).

Semen was collected with an artificial vagina from three fertile bucks, and the pooled ejaculates were washed through ficoll-saline, or conjugated to fluorescein isothiocyanate (FITC) before washing when labelled spermatozoa were required. The methods for FITC-conjugation and washing of spermatozoa have been described previously (Overstreet \& Bedford, 1974). 'Capacitated' spermatozoa were flushed from the uterine horns of fifteen does 12 to $15 \mathrm{hr}$

* Present address: Department of Obstetrics and Gynecology, Cornell University Medical College, New York, N.Y., U.S.A. 
after intravaginal insemination of $0.5 \mathrm{ml}$ washed ejaculated spermatozoa. After concentration of these washings by centrifugation at $2000 \mathrm{rev} / \mathrm{min}(500 \mathrm{~g})$ for $10 \mathrm{~min}$, the number of motile spermatozoa was estimated by multiple haemocytometer counts and a similar number of washed ejaculated spermatozoa was added to the suspension.

In some experiments, spermatozoa were conjugated to FITC before insemination; this resulted in the capacitated sperm population being marked with the fluorescent label. In other experiments, the non-capacitated spermatozoa were conjugated to the fluorochrome immediately before the suspensions were mixed. The mixed suspensions of capacitated and non-capacitated spermatozoa were inseminated in concentrations ranging from $2 \times 10^{4}$ to $13 \times 10^{4}$ motile spermatozoa in $0.1 \mathrm{ml}$ into the tubal ostium of fourteen does $14 \mathrm{hr}$ after an

Table 1. Numbers of spermatozoa associated with eggs recovered after insemination of equal numbers of capacitated and non-capacitated spermatozoa into the oviduct in rabbits

\begin{tabular}{c|c|c|c|c|c}
\hline $\begin{array}{c}\text { Experiment } \\
\text { no. }\end{array}$ & $\begin{array}{c}\text { No. of does } \\
\text { inseminated }\end{array}$ & $\begin{array}{c}\text { No. of } \\
\text { sperm. } \\
\text { inseminated } \\
\left(\times 10^{5}\right)\end{array}$ & $\begin{array}{c}\text { No. of eggs } \\
\text { recovered }\end{array}$ & \multicolumn{2}{|c}{ Mean no. of sperm. legg (range) } \\
\cline { 5 - 6 } & 2 & 10 & 14 & Capacitated & Non-capacitated \\
\hline 1 & 1 & 2 & 5 & $15 \cdot 2(0$ to 25$)$ & $1 \cdot 71(0$ to 4$)$ \\
2 & 2 & 13 & 23 & $8 \cdot 91(2$ to 34$)$ & $2 \cdot 0(1$ to 3$)$ \\
3 & 2 & 5 & 14 & $12 \cdot 3(2$ to 34$)$ & $1 \cdot 39(0$ to 3$)$ \\
4 & 2 & 5 & 19 & $1 \cdot 68(0$ to 5$)$ & $0 \cdot 47(0$ to 2$)$ \\
5 & 1 & 5 & 7 & $24 \cdot 1(7$ to 37$)$ & $3 \cdot 14(1$ to 7$)$ \\
6 & 2 & 30 & 13 & $23 \cdot 2(3$ to 42$)$ & $3 \cdot 0(1$ to 5$)$ \\
7 & 1 & 30 & 9 & $31 \cdot 1(3$ to 75$)$ & $4 \cdot 88(00$ to 9$)$ \\
8 & 1 & 30 & 10 & $33 \cdot 7(12$ to 50$)$ & $3 \cdot 80(2$ to 6$)$ \\
9 & 1 & 30 & & \\
\hline
\end{tabular}

Insemination took place $4 \mathrm{hr}$ after ovulation and eggs were recovered $3 \mathrm{hr}$ after insemination. Either capacitated or non-capacitated spermatozoa were labelled with FITC.

injection of 100 i.u. HCG (Pregnyl, Organon) to induce ovulation. Three hours after insemination, the tubal ova were collected and examined, the number of capacitated and non-capacitated spermatozoa associated with each ovum being assessed on the basis of their fluorescence under u.v. illumination.

An additional experiment was performed to investigate the relative abilities of capacitated and non-capacitated spermatozoa to traverse the barrier presented by the corona radiata. Eggs were recovered 3 to $4 \mathrm{hr}$ after ovulation from twelve donors which had been treated with FSH to induce superovulation (Overstreet \& Bedford, 1974). The cumulus oophorus was dispersed by brief exposure of the eggs to $0.1 \%$ bovine testicular hyaluronidase (Sigma) and the corona radiata was removed from some of the eggs by gentle shaking in $2 \%$ sodium citrate (Harper, 1970). Groups of either corona-invested or denuded eggs were labelled with FITC in the manner previously described (Overstreet, 1973).

The eggs were transferred as a mixed population to the oviducts of ten recipients. Each of these recipient females had been inseminated by way of the tubal ostium with $5 \times 10^{5}$ to $3 \times 10^{7}$ spermatozoa; insemination into one oviduct 


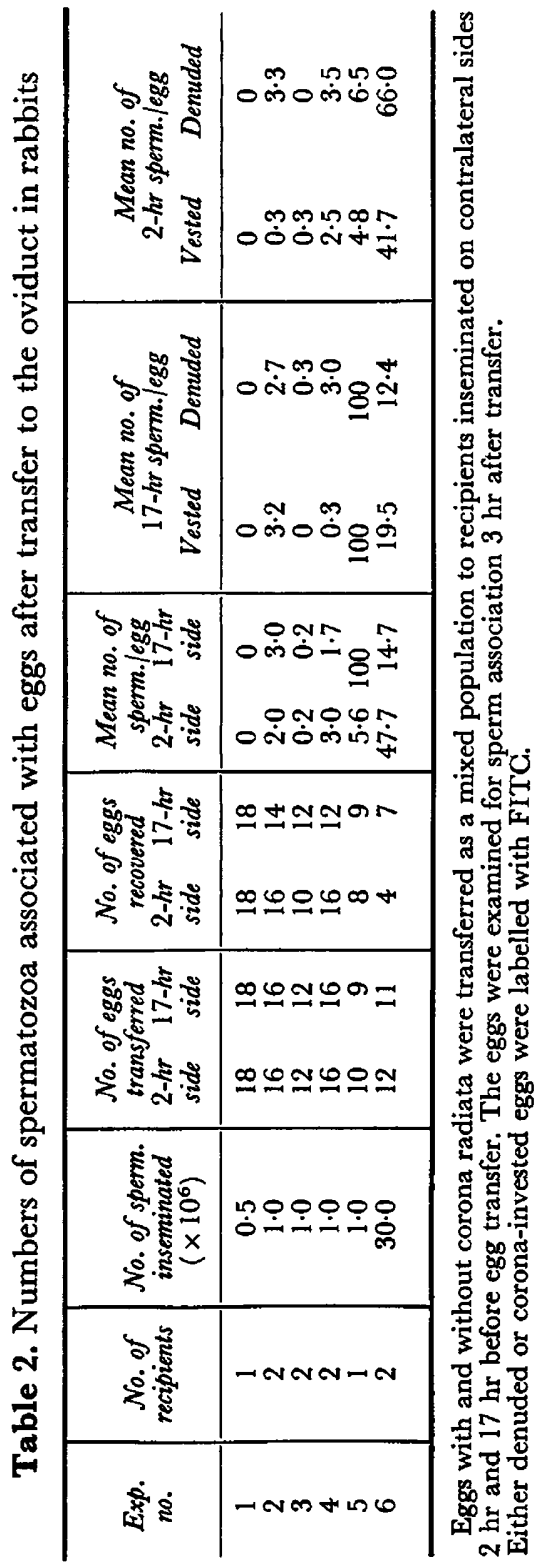


took place $17 \mathrm{hr}$ before egg transfer and, on the contralateral side, $2 \mathrm{hr}$ before transfer. Since capacitation in the oviduct alone requires 10 to $11 \mathrm{hr}$ (Adams \& Chang, 1962), eggs with and without the corona radiata were transferred into the presence of capacitated spermatozoa in one oviduct and non-capacitated spermatozoa in the contralateral oviduct. Both oviducts were flushed $3 \mathrm{hr}$ after egg transfer, and the number of spermatozoa associated with each egg was noted.

In the first series of experiments, intratubal insemination of approximately equal numbers of capacitated and non-capacitated spermatozoa invariably resulted in larger numbers of capacitated spermatozoa being associated with the ova. Following the 3-hr period available for gamete interaction before the eggs were flushed from the oviduct, two to twenty times more capacitated spermatozoa were present on the zonal surface, although non-capacitated spermatozoa were found adhering to some of the eggs in every experiment (Table 1). Ova were recovered in every stage of denudation, and in the majority of experiments at least some of the eggs were completely denuded. There was no apparent relationship, however, between the absence of the granulosa cell investment and the ratio of the two sperm populations at the zonal surface.

Transfer to the oviduct of mixed populations containing eggs with and without the corona radiata resulted in only minor differences in the degree of sperm.egg contact whether the interval between intratubal insemination and egg transfer was $2 \mathrm{hr}$ or $17 \mathrm{hr}$ (Table 2). More spermatozoa tended to be associated with eggs transferred to oviducts containing capacitated spermatozoa, and the number of spermatozoa per egg increased in both groups when a greater concentration of spermatozoa was inseminated.

The present findings strongly suggest that the capacitation of rabbit spermatozoa promotes their interaction with eggs only by facilitating their passage through the granulosa cell investment, and not through change in the adhesive properties of the sperm head. This is essentially the conclusion of Austin (1960) who observed that, following natural mating, spermatozoa are found moving readily between the cells of the granulosa mass but that, in vitro, mature epididymal or ejaculated spermatozoa are unable to penetrate the cumulus oophorus. This difference in the behaviour of capacitated and non-capacitated spermatozoa led Austin to suggest that capacitation allows the release of hyaluronidase from the sperm head, which facilitates its passage to the surface of the zona pellucida. More recently, ultrastructural studies have revealed the means by which enzymes contained in the acrosomal material are released during the acrosome reaction (Barros et al., 1967).

The interaction between ova and non-capacitated rabbit spermatozoa was studied in vivo by Bedford (1967), who counted the number of spermatozoa associated with ova recovered $5 \mathrm{hr}$ after insemination of comparable numbers of epididymal or uterine spermatozoa into contralateral oviducts. The findings led Bedford to conclude that virtually no spermatozoa can establish contact with the zona before capacitation. This influence of capacitation on gamete contact was thought to depend at least in part on an increased facility for passage through the outer egg vestments, but Bedford's experiments could not exclude the possibility that changes in the adhesive properties of the sperm 
head also occurred as a consequence of capacitation. Since, within the limitations of the counting technique, the number of capacitated and non-capacitated spermatozoa in a mixed suspension was equal in the present experiments, a more direct comparison of the level of gamete contact for each sperm population was possible. The differential in numbers of capacitated and non-capacitated spermatozoa is somewhat lower in these experiments than the 16:1 ratio which the group totals of Bedford (1967) suggest, but the approach of noncapacitated spermatozoa to the surface of the zona pellucida in the present experiments may have been facilitated by the action of enzymes released by the capacitated spermatozoa. Nevertheless, the consistent finding of small numbers of non-capacitated spermatozoa at the zonal surface in all experiments indicates the competence of such spermatozoa to stick to the rabbit ovum once they reach its surface, though species differences may occur in this respect. In the hamster, a complex series of reactions has been described between the surfaces of spermatozoa and ova in vitro (Hartmann, Gwatkin \& Hutchinson, 1972; Hartmann \& Hutchinson, 1972), and it has been suggested that alterations in the sperm surface which occur during capacitation facilitate these interactions (Hartmann \& Gwatkin, 1971).

Mixed egg transfer to the ampulla shows here (Table 2) that, after removal of the cumulus oophorus in vitro, non-capacitated $(2 \mathrm{hr})$ spermatozoa are fully competent to establish and maintain contact with the zona pellucida, even in the presence of the corona radiata. Using the electron microscope, Bedford (1969) observed non-capacitated spermatozoa only on denuded areas of the zona $1 \frac{1}{4} \mathrm{hr}$ after tubal insemination of epididymal spermatozoa but following the longer interval in the present experiments, non-capacitated spermatozoa had penetrated between the corona cells of vested eggs to reach the zonal surface.

The data reported here were obtained with the support of NIH grant HD 03623. The technical assistance of Mrs M. J. Bent and Mrs E. Sanidad is gratefully acknowledged. One of us (J.W.O.) is supported by a Medical Scientist Training Fellowship (Grant GM 02042).

\section{REFERENCES}

Adams, C. E. \& Ghang, M. C. (1962) Gapacitation of rabbit spermatozoa in the Fallopian tube and in the uterus. F. exp. Zool. 151, 159.

Austin, C. R. (1960) Gapacitation and the release of hyaluronidase from spermatozoa. F. Reprod. Fert. 1, 310.

Baker, R. D. \& Degen, A. A. (1972) Transport of live and dead boar spermatozoa within the reproductive tract of gilts. F. Reprod. Fert. 28, 369.

Barros, G., Bedford, J. M., Franklin, L. E. \& Austin, G. R. (1967) Membrane vesiculation as a feature of the mammalian acrosome reaction. 7. Cell Biol. 34, C1.

BEDFord, J. M. (1967) Importance of capacitation for establishing contact between eggs and sperm in the rabbit. F. Reprod. Fert. 13, 361.

BEDFord, J. M. (1969) Morphological aspects of sperm capacitation in mammals. Adv. Biosci. 4, 36.

BEDFORD, J. M. (1970) Sperm capacitation and fertilization in mammals. Biol. Reprod., Suppl. 2, 128.

HARPER, M. J. K. (1970) Factors influencing sperm penetration of rabbit eggs in vivo. J. exp. Zool. 173, 47.

Hartmann, J. F. \& Gwatkin, R. B. L. (1971) Alteration of sites on the mammalian sperm surface following capacitation. Nature, Lond. 25, 479. 
Hartmann, J. F., Gwatkin, B. L. \& Hutchison, C. F. (1972) Early contact interactions between mammalian gametes in vitro: evidence that the vitellus influences adherence between the sperm and zona pellucida. Proc. natn. Acad. Sci. U.S.A. 69, 2767.

Hartmann, J. F. \& Hutchison, G. F. (1972) Nature of the pre-penetration contact interactions between gametes of the golden hamster in vitro. F. Cell. Biol. 55, 107a.

MeLLISH, K. S. \& BAKER, R. D. (1970) Marking boar spermatozoa with fluorochromes for evaluating spermatozoan transport within gilts. J. Anim. Sci. 31, 917.

Overstreet, J. W. (1973) The labelling of living rabbit ova with fluorescent dyes. F. Reprod. Fert. 32, 291.

Overstreet, J. W. \& Adams, G. E. (1971) Mechanisms of selective fertilization in the rabbit: sperm transport and viability. 7. Reprod. Fert. 26, 219.

Overstreet, J. W. \& Bedford, J. M. (1974) Transport, capacitation and fertilizing ability of epididymal spermatozoa. F. exp. Zool. (in press).

Thibault, G. (1971) Normal and abnormal fertilization in mammals. Adv. Biosci. 6, 63.

Yanagimachi, R. \& Noda, Y. D. (1970) Physiological changes in the post nuclear cap region of mammalian spermatozoa: a necessary preliminary to the membrane fusion between sperm and egg cells. F. Ultrastruct. Res. 31, 486. 